\title{
New Antibiotics Miyakamides Produced by a Fungus
}

\author{
Kazuro Shiomi, ${ }^{*}$ Kenji Hatae, ${ }^{\dagger}$ Yuichi Yamaguchi, Rokuro Masuma, \\ Hiroshi Tomoda, Susumu KobaYASHI ${ }^{\dagger}$ and SATOSHI ŌMURA* \\ Kitasato Institute for Life Sciences, Kitasato University, and The Kitasato Institute, \\ 5-9-1 Shirokane, Minato-ku, Tokyo 108-8641, Japan \\ ${ }^{\dagger}$ Faculty of Pharmaceutical Sciences, Science University of Tokyo, \\ 12 Ichigaya-Funagawara-Machi, Shinjuku-ku, Tokyo 162-0826, Japan
}

(Received for publication May 7, 2002)

\begin{abstract}
New antibiotics, miyakamides $A_{1}, A_{2}, B_{1}$, and $B_{2}$, were isolated from the cultured broth of Aspergillus flavus Link var. columnaris FKI-0739 together with known compounds, parasiticolide A, hydroxyaspergillic acid, and kojic acid. The structure of miyakamide $\mathrm{A}_{1}$ is $N$ acetyl-L-phenylalanyl- $N$-methyl-L-phenylalanyl- $(\alpha Z)-\alpha, \beta$-didehydrotryptamine, and miyakamide $\mathrm{A}_{2}$ is $E$ isomer of $\mathrm{A}_{1}$ at didehydrotryptamine. The structure of miyakamide $\mathrm{B}_{1}$ is $N$-acetyl-Ltyrosyl- $N$-methyl-L-phenylalanyl-( $\alpha Z)-\alpha, \beta$-didehydrotryptamine, and $\mathrm{B}_{2}$ is $E$ isomer of $\mathrm{B}_{1}$. Both miyakamides $A_{1}$ and $B_{1}$ existed as equilibrium isomers in solvents, and this isomerism was associated with cis-trans rotation of the amide bond between two amino acids. Conformational isomerism between two amino acids of miyakamides $\mathrm{A}_{2}$ and $\mathrm{B}_{2}$ is cis-form. Miyakamides showed growth inhibitory activity against brine shrimp, Artemia salina.
\end{abstract}

In the course of screening for insecticidal and nematocidal antibiotics, we have isolated some new antibiotics from microbial metabolites. ${ }^{1 \sim 5)}$ Our continuous screening efforts to find antibiotics were rewarded by the finding of new antibiotics, miyakamides $A_{1}, A_{2}, B_{1}$, and $B_{2}$ (1 4, Fig. 1), which were isolated from a cultured broth of Aspergillus sp. FKI-0739. In this report, we describe taxonomy of the producing strains and fermentation, isolation, structure elucidation, and biological properties of miyakamides.

\section{Results and Discussion}

Taxonomy of Producing Strain FKI-0739

Strain FKI-0739 was originally isolated from a fallen leaf collected at Miyakojima Island, Japan. For the taxonomic studies of the fungus, Czapek yeast extract agar (CYA), malt extract agar (MEA), and Czapek yeast extract agar with $20 \%$ sucrose (CY20S) were used. Colonies on CYA were $55 \sim 60 \mathrm{~mm}$ in diameter after 7 days at $25^{\circ} \mathrm{C}$, floccose to farinaceous, and light olive (1 $1 / 2$ ie) to golden olive (1 $1 / 2 \mathrm{lg}$ ) in color with abundant sporulation; the reverse was dusty yellow (1 $1 / 2 \mathrm{gc}$ ). Colonies on MEA were $57 \sim 63 \mathrm{~mm}$ in diameter after 7 days at $25^{\circ} \mathrm{C}$, floccose to farinaceous, and butter yellow (1 $1 / 2 \mathrm{ga}$ ) in color with abundant sporulation; the reverse was white to cream (1 $1 / 2$ ca). Colonies on CY20S were $53 \sim 55 \mathrm{~mm}$ in diameter after 7 days at $25^{\circ} \mathrm{C}$, floccose to farinaceous, and olive gray (1 ig) in color with abundant sporulation; the reverse was white to light yellow (1 1/2 ea). Morphological properties were observed after 7 days on CYA under a microscope and a scanning electron microscope (Fig. 2). Conidial heads were loosely columnar up to $500 \mu \mathrm{m}$. Conidiophores were born from the substratum and aerial hyphae and 400 $1,000 \times 3.5 \sim 5.0 \mu \mathrm{m}$ in diameter. They were almost roughwalled and hyaline to slightly brown. Vesicles were clavate to subglobose, $30 \sim 60 \mu \mathrm{m}$ in diameter, and fertile over the upper one-half of their surface. Phialides were consistently in a single series, ampulliform, closely packed, $6 \sim 11 \times$ $3 \sim 5 \mu \mathrm{m}$ in size, and producing conidia in long chain. Conidia were globose to subglobose, $3.5 \sim 5.5 \mu \mathrm{m}$ in diameter, conspicuously echinulate, and olive-brown in mass.

\footnotetext{
* Corresponding author: shiomik@pharm.kitasato-u.ac.jp; omura-s@kitasato.or.jp
} 
Fig. 1. Structures of miyakamides $A_{1}(1), A_{2}(2), B_{1}(3)$, and $B_{2}$ (4).

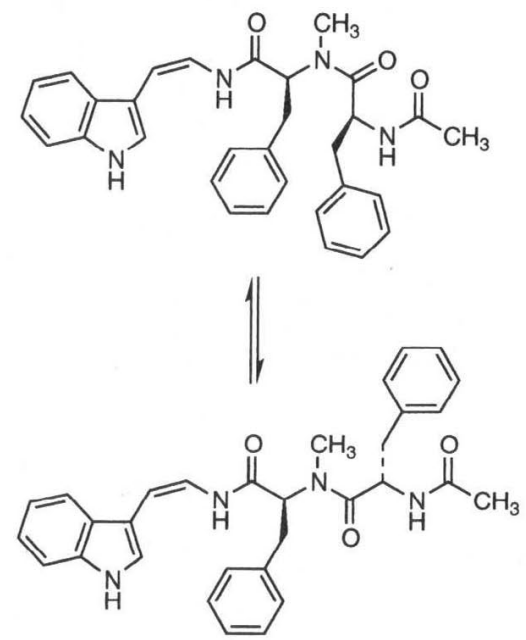

Miyakamide $\mathrm{A}_{1}(\mathbf{1})$

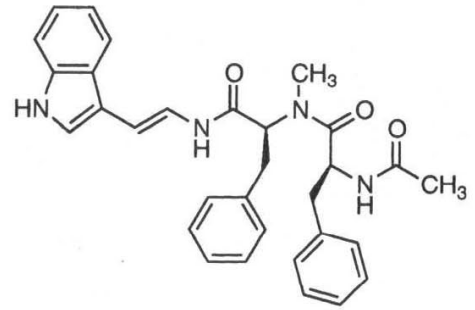

Miyakamide $\mathrm{A}_{2}$ (2)

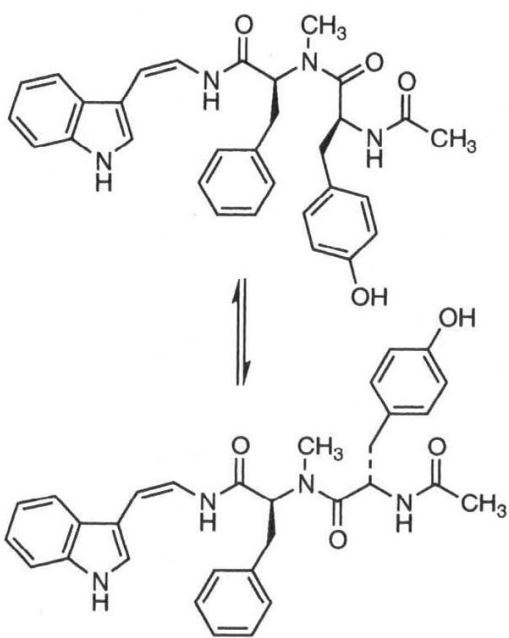

Miyakamide $\mathrm{B}_{1}(\mathbf{3})$

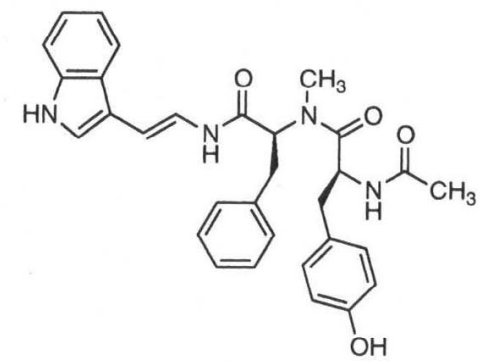

Miyakamide $\mathrm{B}_{2}(\mathbf{4})$

Fig. 2. Scanning electron micrograph of strain FKI-0739.

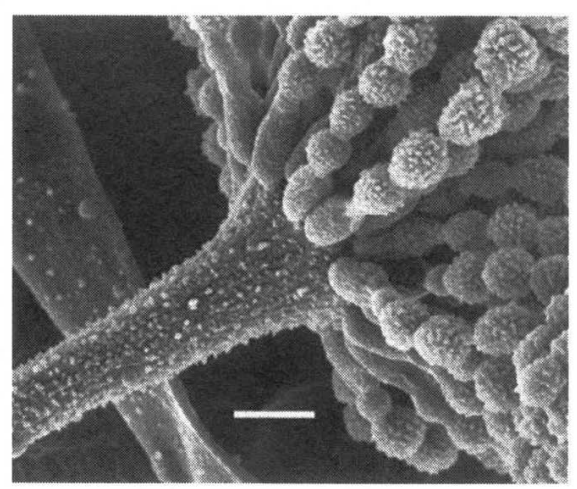

Bar represents $5 \mu \mathrm{m}$. dextrose agar was inoculated into a large test tube containing $10 \mathrm{ml}$ of a seed medium and incubated on a reciprocal shaker at $27^{\circ} \mathrm{C}$ for 3 days. One milliliter of the seed culture was transferred into each of five $500-\mathrm{ml}$ Erlenmeyer flasks containing $100 \mathrm{ml}$ of a production 
medium. The fermentation was carried out on a rotary shaker $(210 \mathrm{rpm})$ at $27^{\circ} \mathrm{C}$ for 6 days.

\section{Isolation}

The cultured broth $(500 \mathrm{ml})$ was centrifuged and the supernatant was extracted with ethyl acetate. The mycelia were extracted with methanol, which was then removed from the extract by evaporation. The aqueous extract was partitioned with ethyl acetate, and the organic layer was combined with the supernatant extract and concentrated to dryness in vacuo to afford a crude material $(388 \mathrm{mg})$. It was chromatographed over a silica gel column. The eluate of $\mathrm{CHCl}_{3}$ was concentrated to yield $7.6 \mathrm{mg}$ of yellow powder, which was identified as parasiticolide A (astellolide A, 5). ${ }^{8}$ ) Active fractions eluted with $\mathrm{CHCl}_{3}$ - methanol (100: 1) and $\mathrm{CHCl}_{3}$ - methanol $(50: 1)$ were concentrated to yield crude miyakamides A $(258 \mathrm{mg})$ and $\mathrm{B}(18.8 \mathrm{mg})$, respectively. The $\mathrm{CHCl}_{3}$-methanol eluate $(10: 1)$ was concentrated $(32.8 \mathrm{mg})$ and further purified by Sephadex LH-20 chromatography to yield an orange powder (18.4 $\mathrm{mg}$ ) of hydroxyaspergillic acid ${ }^{10)}(6)$ and a white powder $(2.3 \mathrm{mg})$ of kojic acid ${ }^{11)}(7)$.

The crude miyakamide A was purified by HPLC under the following conditions: column, Senshu pak Pegasil ODS (i.d. $20 \times 250 \mathrm{~mm}$, Senshu Scientific Co.); mobile phase, $60 \% \mathrm{CH}_{3} \mathrm{CN}$; flow rate, $7 \mathrm{ml} /$ minute; detection, multi- wavelength detector MD-910 (JASCO). Miyakamides $\mathrm{A}_{1}$ (1) and $\mathrm{A}_{2}$ (2) were eluted at 28.0 and 22.7 minutes, respectively, under the above conditions. The HPLC eluates were concentrated to remove $\mathrm{CH}_{3} \mathrm{CN}$, extracted with ethyl acetate, and concentrated to dryness to give pale yellow powders of 1 (220 mg) and $2(10.2 \mathrm{mg})$. The crude miyakamide B was purified by HPLC using the same condition as miyakamide A except that the mobile phase was $40 \% \mathrm{CH}_{3} \mathrm{CN}$ with $0.05 \%$ phosphate buffered saline, $\mathrm{pH}$ 7.0. Pale yellow powders of miyakamides $\mathrm{B}_{1}(3,2.0 \mathrm{mg})$ and $B_{2}(4,2.9 \mathrm{mg})$ were obtained from the eluate at 79.7 and 63.1 minutes, respectively.

\section{Physico-chemical Properties}

Physico-chemical properties of miyakamides are summarized in Table 1. They showed similar UV spectra. The common IR absorbances observed at $1630 \sim 1650 \mathrm{~cm}^{-1}$ suggested the presence of amide carbonyl groups in their structures. The molecular formulae of $\mathbf{1}$ and $\mathbf{2}$ were established as $\mathrm{C}_{31} \mathrm{H}_{32} \mathrm{~N}_{4} \mathrm{O}_{3}$ and the molecular formulae of 3 and 4 were established as $\mathrm{C}_{31} \mathrm{H}_{32} \mathrm{~N}_{4} \mathrm{O}_{4}$ by HR-FAB-MS. Chemical shifts in the ${ }^{1} \mathrm{H}$ and ${ }^{13} \mathrm{C}$ NMR of $\mathbf{1} \sim \mathbf{4}$ are shown in Tables 2 and 3. Miyakamides showed positive color reaction by Rydon-Smith reagent. As ${ }^{13} \mathrm{C}$ NMR assignment of 6 has not been reported, its NMR data are shown in the experimental section.

Table 1. Physico-chemical properties of miyakamides $A \sim D(1 \sim 4)$.

\begin{tabular}{|c|c|c|c|c|c|}
\hline & & Miyakamide $A_{1}$ (1) & Miyakamide $\mathrm{A}_{2}$ (2) & Miyakamide $\mathbf{B}_{1}$ (3) & Miyakamide $B_{2}$ (4) \\
\hline \multicolumn{2}{|l|}{ Appearance } & Pale yellow powder & Pale yellow powder & Pale yellow powder & Pale yellow powder \\
\hline \multicolumn{2}{|l|}{ Melting point } & 92-94 (decomp.) & $98-100^{\circ}$ (decomp.) & $103-106^{\circ}$ (decomp.) & $107-111^{\circ}$ (decomp.) \\
\hline \multicolumn{2}{|l|}{$[\alpha]_{D}^{25}$} & $-34.9^{\circ}(c 0.230, \mathrm{MeOH})$ & $-27.1^{\circ}(c 0.244, \mathrm{MeOH})$ & $-24.2^{\circ}(c 0.153, \mathrm{MeOH})$ & $-34.1^{\circ}(c 0.291, \mathrm{MeOH})$ \\
\hline \multicolumn{2}{|c|}{ Molecular formula } & $\mathrm{C}_{31} \mathrm{H}_{32} \mathrm{~N}_{4} \mathrm{O}_{3}$ & $\mathrm{C}_{31} \mathrm{H}_{32} \mathrm{~N}_{4} \mathrm{O}_{3}$ & $\mathrm{C}_{31} \mathrm{H}_{32} \mathrm{~N}_{4} \mathrm{O}_{4}$ & $\mathrm{C}_{31} \mathrm{H}_{32} \mathrm{~N}_{4} \mathrm{O}_{4}$ \\
\hline \multicolumn{2}{|c|}{ Molecular weight } & 508.6 & 508.6 & 524.6 & 524.6 \\
\hline \multicolumn{2}{|c|}{ FAB-MS $(m / z)$ Positive: } & $508[\mathrm{M}]^{+}, 531[\mathrm{M}+\mathrm{Na}]^{+}$ & $508[\mathrm{M}]^{+}, 531[\mathrm{M}+\mathrm{Na}]^{+}$ & $524[\mathrm{M}]^{+}, 547[\mathrm{M}+\mathrm{Na}]^{+}$ & $524[\mathrm{M}]^{+}, 547[\mathrm{M}+\mathrm{Na}]^{+}$ \\
\hline \multicolumn{3}{|c|}{ Negative: $507[\mathrm{M}-\mathrm{H}]^{-}$} & $507[\mathrm{M}-\mathrm{H}]^{-}$ & $523[\mathrm{M}-\mathrm{H}]^{-}$ & $523[\mathrm{M}-\mathrm{H}]^{-}$ \\
\hline \multicolumn{2}{|c|}{ HR-FAB-MS $(m / z)$} & $\mathrm{C}_{31} \mathrm{H}_{31} \mathrm{~N}_{4} \mathrm{O}_{3}[\mathrm{M}-\mathrm{H}]^{-}$ & $\mathrm{C}_{31} \mathrm{H}_{32} \mathrm{~N}_{4} \mathrm{O}_{3} \mathrm{Na}[\mathrm{M}+\mathrm{Na}]^{+}$ & $\mathrm{C}_{31} \mathrm{H}_{31} \mathrm{~N}_{4} \mathrm{O}_{4}[\mathrm{M}-\mathrm{H}]^{-}$ & $\mathrm{C}_{31} \mathrm{H}_{31} \mathrm{~N}_{4} \mathrm{O}_{4}[\mathrm{M}-\mathrm{H}]^{-}$ \\
\hline & Calcd: & 507.2396 & 531.2372 & 523.2345 & 523.2345 \\
\hline & Found: & 507.2373 & 531.2363 & 523.2337 & 523.2347 \\
\hline \multicolumn{2}{|c|}{$\mathrm{UV} \lambda_{\max }^{\mathrm{MeOH}} \mathrm{nm}(\varepsilon)$} & $\begin{array}{l}212(\mathrm{sh}, 8,600), 228(13,600) \\
284(\mathrm{sh}, 8,200), 288(\mathrm{sh}, \\
9,000), 300(10,400)\end{array}$ & $\begin{array}{l}204(25,900), 258(5,800) \\
280(6,100), 306(6,400)\end{array}$ & $\begin{array}{l}204(37,700), 225(29,300) \\
288(15,200), 301(\mathrm{sh}, \\
14,150)\end{array}$ & $\begin{array}{l}202(26,700), 225(18,860), \\
280(\text { sh, } 10,500), 307 \\
(13,100)\end{array}$ \\
\hline \multicolumn{2}{|c|}{ IR $v_{\max }(\mathrm{KBr}) \mathrm{cm}^{-1}$} & $\begin{array}{l}3307,2927,1651,1633, \\
1538,1496,1456,1230, \\
1103,748,700\end{array}$ & $\begin{array}{l}3419,2962,2926,2854 \\
1628,1549,1454,1261 \\
1097,1029,800,743,700\end{array}$ & $\begin{array}{l}3417,2925,1653,1633, \\
1537,1497,1456,1230, \\
1103,744,700\end{array}$ & $\begin{array}{l}3415,2923,1651,1633 \\
1552,1516,1456,1230 \\
1101,947,743,702\end{array}$ \\
\hline \multirow[t]{2}{*}{ Solubility } & Soluble: & $\begin{array}{l}\text { Acetone, } \mathrm{MeOH}, \mathrm{EtOAc} \text {, } \\
\text { Acetonitrile, } \mathrm{CHCl}_{3}\end{array}$ & $\begin{array}{l}\text { Acetone, } \mathrm{MeOH}, \mathrm{EtOAc}, \\
\text { Acetonitrile, } \mathrm{CHCl}_{3}\end{array}$ & $\begin{array}{l}\text { Acetone, } \mathrm{MeOH}, \mathrm{EtOAc} \text {, } \\
\text { Acetonitrile, } \mathrm{CHCl}_{3}\end{array}$ & $\begin{array}{l}\text { Acetone, } \mathrm{MeOH}, \mathrm{EtOAc} \text {, } \\
\text { Acetonitrile, } \mathrm{CHCl}_{3}\end{array}$ \\
\hline & Insoluble: & $: \mathrm{H}_{2} \mathrm{O}, n$-Hexane & $\mathrm{H}_{2} \mathrm{O}, n$-Hexane & $\mathrm{H}_{2} \mathrm{O}, n$-Hexane & $\mathrm{H}_{2} \mathrm{O}, n$-Hexane \\
\hline Color reaction & Positive: & $5 \% \mathrm{H}_{2} \mathrm{SO}_{4}$, Rydon-Smith & $5 \% \mathrm{H}_{2} \mathrm{SO}_{4}$, Rydon-Smith & $5 \% \mathrm{H}_{2} \mathrm{SO}_{4}$, Rydon-Smith & $5 \% \mathrm{H}_{2} \mathrm{SO}_{4}$, Rydon-Smith \\
\hline
\end{tabular}


Table 2. ${ }^{1} \mathrm{H}$ and ${ }^{13} \mathrm{C}$ NMR data of miyakamides $\mathrm{A}_{1}(\mathbf{1})$ and $\mathrm{A}_{2}$ (2) (in acetone- $d_{6}$ ).

\begin{tabular}{|c|c|c|c|c|c|c|}
\hline & \multicolumn{2}{|c|}{ cis-amide } & \multicolumn{2}{|c|}{ trans-amide } & \multicolumn{2}{|c|}{ Miyakamide $\mathrm{A}_{2}(\mathbf{2})$} \\
\hline & ${ }^{13} \mathrm{C}(\mathrm{ppm})$ & ${ }^{1} \mathbf{H}(\mathrm{ppm})^{a}$ & ${ }^{13} \mathrm{C}(\mathrm{ppm})$ & ${ }^{1} \mathrm{H}(\mathrm{ppm})^{a}$ & ${ }^{13} \mathrm{C}$ (ppm) & ${ }^{1} \mathrm{H}(\mathrm{ppm})^{a}$ \\
\hline $\begin{array}{l}\text { AcPhe Ac }(\mathrm{Me}) \\
\text { AcPhe Ac }(\mathrm{C}=\mathrm{O})\end{array}$ & $21.7 \mathrm{q}$ & $1.49 \mathrm{~s}$ & $22.6 \mathrm{q}$ & $1.79 \mathrm{~s}$ & $22.5 \mathrm{q}$ & $1.92 \mathrm{~s}$ \\
\hline AcPhe NH & & $7.65 \mathrm{~m}$ & & $7.16 \mathrm{~m}$ & & $7.81 \mathrm{~d}(6.6)$ \\
\hline $\begin{array}{l}\text { AcPhe } \mathrm{C}=\mathrm{O} \\
\text { AcPhe } \alpha\end{array}$ & $172.8 \mathrm{~s}$ & & $174.0 \mathrm{~s}$ & & $172.3 \mathrm{~s}$ & \\
\hline $\begin{array}{l}\text { AcPhe } \alpha \\
\text { AcPhe } \beta\end{array}$ & $\begin{array}{l}51.0 \mathrm{~d} \\
37.6 \mathrm{t}\end{array}$ & $\begin{array}{l}4.74 \text { ddd }(9.1,6.7,6.1) \\
2.14 \text { dd }(13.8,6.1) \\
2.70 \text { dd }(13.8,9.1)\end{array}$ & $\begin{array}{l}51.4 \mathrm{~d} \\
39.1 \mathrm{t}\end{array}$ & $\begin{array}{l}5.06 \mathrm{ddd}(7.8,7.8,7.8) \\
2.80 \mathrm{~m} \\
2.88 \mathrm{~m}\end{array}$ & $\begin{array}{l}50.8 \mathrm{~d} \\
37.3 \mathrm{t}\end{array}$ & $\begin{array}{l}4.90 \mathrm{ddd}(10.2,6.6,4.7) \\
1.78 \mathrm{dd}(13.9,4.7), \\
2.52 \mathrm{dd}(13.9,10.2)\end{array}$ \\
\hline AcPhe 1 & $138.2 \mathrm{~s}$ & & $138.0 \mathrm{~s}$ & & $138.1 \mathrm{~s}$ & \\
\hline AcPhe 2,6 & $130.14 \mathrm{dx} 2$ & $7.11 \mathrm{~m}$ & $130.12 \mathrm{dx} 2$ & $7.11 \mathrm{~m}$ & $130.1 \mathrm{dx} 2$ & $7.19 \mathrm{~m}$ \\
\hline AcPhe 3,5 & $129.5 \mathrm{dx} 2$ & $7.25 \mathrm{~m}$ & $129.1 \mathrm{dx} 2$ & $7.15 \mathrm{~m}$ & $129.1 \mathrm{dx} 2$ & $7.28 \mathrm{~m}$ \\
\hline AcPhe 4 & $127.34 \mathrm{~d}$ & $7.17 \mathrm{~m}$ & $127.29 \mathrm{~d}$ & $7.10 \mathrm{~m}$ & $127.37 \mathrm{~d}$ & $7.18 \mathrm{~m}$ \\
\hline MePhe NMe & $29.7 \mathrm{q}$ & $2.94 \mathrm{~s}$ & $31.9 \mathrm{q}$ & $2.82 \mathrm{~s}$ & $29.3 \mathrm{q}$ & $2.87 \mathrm{~s}$ \\
\hline $\begin{array}{l}\text { MePhe C=O } \\
\text { MePhe } \alpha\end{array}$ & $\begin{array}{r}167.8 \mathrm{~s} \\
62.4 \mathrm{~d}\end{array}$ & $5.48 \mathrm{dd}(8.7,6.6)$ & $\begin{array}{r}167.7 \mathrm{~s} \\
59.5 \mathrm{~d}\end{array}$ & $5.31 \mathrm{dd}(8.7,6.9)$ & $\begin{array}{r}166.6 \mathrm{~s} \\
632 \mathrm{~d}\end{array}$ & $5.21 \mathrm{dd}(9.4,5.2)$ \\
\hline MePhe $\beta$ & $\begin{array}{l}02.4 \mathrm{a} \\
35.7 \mathrm{t}\end{array}$ & $\begin{array}{l}2.55 \mathrm{dd}(13.9,8.7) \\
3.24 \mathrm{dd}(13.9,6.6)\end{array}$ & $34.0 \mathrm{t}$ & $\begin{array}{l}3.04 \text { dd }(14.6,8.7) \\
3.31 \mathrm{dd}(14.6,6.9)\end{array}$ & $34.9 \mathrm{t}$ & $\begin{array}{l}2.74 \text { dd }(14.0,9.4) \\
3.26 \text { dd }(14.0,5.2)\end{array}$ \\
\hline MePhe 1 & $138.9 \mathrm{~s}$ & & $138.5 \mathrm{~s}$ & & $139.3 \mathrm{~s}$ & \\
\hline MePhe 2,6 & $130.5 \mathrm{dx} 2$ & $7.31 \mathrm{~m}$ & $129.9 \mathrm{dx} 2$ & $7.23 \mathrm{~m}$ & $130.5 \mathrm{dx} 2$ & $7.37 \mathrm{~d}(7.1)$ \\
\hline MePhe 3,5 & $129.0 \mathrm{dx} 2$ & $7.26 \mathrm{~m}$ & $129.2 \mathrm{dx} 2$ & $7.25 \mathrm{~m}$ & $129.5 \mathrm{dx} 2$ & $7.24 \mathrm{~m}$ \\
\hline MePhe 4 & $127.4 \mathrm{~d}$ & $7.13 \mathrm{~m}$ & $127.25 \mathrm{~d}$ & $7.19 \mathrm{~m}$ & $127.41 \mathrm{~d}$ & $7.14 \mathrm{~m}$ \\
\hline $\operatorname{Tra~NH}^{b}$ & & $9.18 \mathrm{~d}(10.2)$ & & $8.47 \mathrm{~d}(10.8)$ & & $10.11 \mathrm{~d}(10.0)$ \\
\hline dhTra $\alpha$ & $119.5 \mathrm{~d}$ & $6.79 \mathrm{dd}(10.2,9.6)$ & $119.5 \mathrm{~d}$ & $6.84 \mathrm{dd}(10.8,9.4)$ & $121.0 \mathrm{~d}$ & $7.46 \mathrm{dd}(14.9,10.0)$ \\
\hline dhTra $\beta$ & $104.5 \mathrm{~d}$ & $5.98 \mathrm{~d}(9.6)$ & $103.1 \mathrm{~d}$ & $6.05 \mathrm{~d}(9.4)$ & $107.7 \mathrm{~d}$ & $6.56 \mathrm{~d}(14.9)$ \\
\hline dhTra 1 & & 10.4 br.s & & 10.5 br.s & & $10.24 \mathrm{~s}$ \\
\hline dhTra 2 & $124.9 \mathrm{~d}$ & $7.66 \mathrm{~d}(2.5)$ & $123.7 \mathrm{~d}$ & $7.58 \mathrm{~d}(2.5)$ & $123.8 \mathrm{~d}$ & $7.36 \mathrm{~d}(2.2)$ \\
\hline dhTra 3 & $111.1 \mathrm{~s}$ & & $111.5 \mathrm{~s} \mathrm{~d}$ & & $113.5 \mathrm{~s}$ & \\
\hline dhTra 3a & $128.0 \mathrm{~s}$ & & $127.9 \mathrm{~s}$ & & $126.4 \mathrm{~s}$ & \\
\hline dhTra 4 & $119.3 \mathrm{~d}$ & $7.56 \mathrm{~d}(8.0)$ & $119.3 \mathrm{~d}$ & $7.64 \mathrm{~d}(7.7)$ & $120.2 \mathrm{~d}$ & $7.73 \mathrm{~d}(7.9)$ \\
\hline dhTra 5 & $120.2 \mathrm{~d}$ & $7.06 \mathrm{~m}$ & $120.3 \mathrm{~d}$ & $7.09 \mathrm{~m}$ & $120.3 \mathrm{~d}$ & 7.09 dd $(7.9,7.4)$ \\
\hline dhTra 6 & $122.6 \mathrm{~d}$ & $7.11 \mathrm{~m}$ & $123.0 \mathrm{~d}$ & $7.16 \mathrm{~m}$ & $122.6 \mathrm{~d}$ & $7.12 \mathrm{dd}(8.3,7.4)$ \\
\hline dhTra 7 & $112.1 \mathrm{~d}$ & $7.38 \mathrm{~d}(7.2)$ & $112.3 \mathrm{~d}$ & $7.44 \mathrm{~d}(8.3)$ & $112.5 \mathrm{~d}$ & $7.40 \mathrm{~d}(8.3)$ \\
\hline dhTra $7 a$ & $137.0 \mathrm{~s}$ & & $137.1 \mathrm{~s}$ & & $138.2 \mathrm{~s}$ & \\
\hline
\end{tabular}

$a$ : The coupling constants are in parentheses. $b$ : dhTra is $\alpha, \beta$-didehydrotryptamine.

\section{Structure Elucidation}

The structure of $\mathbf{2}$ was elucidated by NMR study. Analysis of the ${ }^{1} \mathrm{H}$ NMR, ${ }^{13} \mathrm{C}$ NMR, DEPT, and HMQC spectra revealed the presence of eight quaternary, nineteen methine, two methylene, and two methyl carbons. Aromatic protons were assigned as two monosubstituted benzenes and one 1,2-disubstituted benzene, and they were deduced to be two phenylalanines and one indole, respectively, by ${ }^{1} \mathrm{H}-{ }^{1} \mathrm{H}-\mathrm{COSY}$ and $\mathrm{HMBC}$ (Fig. 3). ${ }^{1} \mathrm{H}-{ }^{13} \mathrm{C}$ long-range couplings by $\mathrm{HMBC}$ indicated that the amino residue of one phenylalanine is acetylated (AcPhe) and the other is methylated (MePhe). A partial structure $-\mathrm{CH}=\mathrm{CH}-\mathrm{NH}-$ is connected to $\mathrm{C}-3$ of the indole by ${ }^{1} \mathrm{H}-{ }^{13} \mathrm{C}$ long-range couplings, which forms $\alpha, \beta$-didehydrotryptamine (dhTra). ${ }^{1} \mathrm{H}-{ }^{13} \mathrm{C}$ long-range couplings between MePhe $\alpha\left(\delta_{\mathrm{H}} 5.20\right)$ and $\mathrm{AcPhe} \mathrm{C}=\mathrm{O}\left(\delta_{\mathrm{C}} 172.3\right)$ and between MePhe NMe $\left(\delta_{\mathrm{H}}\right.$ 2.87) and AcPhe $\mathrm{C}=\mathrm{O}$ proved the connection of AcPheMePhe. ${ }^{1} \mathrm{H}-{ }^{13} \mathrm{C}$ long-range couplings between $\operatorname{dhTra} \alpha\left(\delta_{\mathrm{H}}\right.$ 7.46) and MePhe $\mathrm{C}=\mathrm{O}\left(\delta_{\mathrm{C}} 166.6\right)$ and between dhTra NH
( $\delta_{\mathrm{H}}$ 10.10) and MePhe $\mathrm{C}=\mathrm{O}$ indicated the connection of MePhe-dhTra. Thus the planar structure of $\mathbf{2}$ was elucidated as AcPhe-MePhe-dhTra. NOESY experiment indicated that the conformational isomerism of the amide bond between two phenylalanines is cis by NOE between AcPhe $\alpha-\mathrm{H}\left(\delta_{\mathrm{H}}\right.$ 4.90) and MePhe $\alpha-\mathrm{H}$ (Fig. 3). The geometrical isomerism between $\alpha$ and $\beta$ methines of dhTra was elucidated as $E$ by the vicinal coupling constants of the methine protons $(J=14.9 \mathrm{~Hz})$ and NOEs between $\operatorname{dhTra} \beta\left(\delta_{\mathrm{H}} 6.56\right) / \mathrm{dhTra}$ NH, dhTra $\beta /$ dhTra $2\left(\delta_{\mathrm{H}} 7.36\right)$, and dhTra $\alpha /$ dhTra $4\left(\delta_{\mathrm{H}}\right.$ 7.73). The chirality of each amino acid was elucidated by chiral HPLC of acid hydrolysate of 2 , which revealed the presence of $1 \mathrm{~mol}$ of L-phenylalanine and $N$-methyl-Lphenylalanine. Consequently, the structure of $\mathbf{2}$ was elucidated as $N$-acetyl-L-phenylalanyl- $N$-methyl-L-phenylalanyl- $(\alpha E)-\alpha, \beta$-didehydrotryptamine in which the amide bond between $N$-acetylphenylalanine and $N$-methylphenylalanine is cis.

The molecular formula of $\mathbf{1}$ was the same as that of $\mathbf{2}$. More than $90 \%$ of $\mathbf{1}$ was converted to $\mathbf{2}$ in methanol at 
Table 3. ${ }^{1} \mathrm{H}$ and ${ }^{13} \mathrm{C}$ NMR data of miyakamides $\mathrm{B}_{1}(3)$ and $\mathrm{B}_{2}(4)$ (in acetone- $d_{6}$ ).

\begin{tabular}{|c|c|c|c|c|c|c|}
\hline & \multicolumn{2}{|c|}{ cis-amide } & \multicolumn{2}{|c|}{ trans-amide } & \multicolumn{2}{|c|}{ Miyakamide $B_{2}(4)$} \\
\hline & ${ }^{13} \mathrm{C}$ (ppm) & ${ }^{1} \mathrm{H}(\mathrm{ppm})^{a}$ & ${ }^{13} \mathrm{C}(\mathrm{ppm})$ & ${ }^{1} \mathrm{H}(\mathrm{ppm})^{a}$ & ${ }^{13} \mathrm{C}$ (ppm) & ${ }^{1} \mathrm{H}(\mathrm{ppm})^{a}$ \\
\hline AcTyr Ac (Me) & $21.8 \mathrm{q}$ & $1.52 \mathrm{~s}$ & $22.6 \mathrm{q}$ & $1.80 \mathrm{~s}$ & $22.6 \mathrm{q}$ & $1.94 \mathrm{~s}$ \\
\hline AcTyr Ac $(C=O)$ & $171.8 \mathrm{~s}$ & & $169.3 \mathrm{~s}$ & & $172.2 \mathrm{~s}$ & \\
\hline AcTyr NH & & $7.60 \mathrm{~d}(7.3)$ & & $7.12 \mathrm{~m}$ & & $7.77 \mathrm{~d}(7.7)$ \\
\hline AcTyr $\mathrm{C}=\mathrm{O}$ & $172.9 \mathrm{~s}$ & & $174.3 \mathrm{~s}$ & & $172.9 \mathrm{~s}$ & \\
\hline AcTyr $\alpha$ & $51.5 \mathrm{~d}$ & 4.67 ddd $(8.8,7.3,6.3)$ & $51.7 \mathrm{~d}$ & 4.98 ddd $(7.7,7.7,7.7)$ & $51.2 \mathrm{~d}$ & 4.84 ddd $(9.9,6.1,5.3)$ \\
\hline AcTyr $\beta$ & $37.0 \mathrm{t}$ & $\begin{array}{l}2.15 \mathrm{dd}(13.8,6.3) \\
2.61 \mathrm{dd}(13.8,8.8)\end{array}$ & $38.5 \mathrm{t}$ & $\begin{array}{l}2.71 \mathrm{~m} \\
2.80 \mathrm{~m}\end{array}$ & $36.6 \mathrm{t}$ & $\begin{array}{l}1.80 \text { dd }(13.9,5.3) \\
2.46 \text { dd }(13.9,9.9)\end{array}$ \\
\hline AcTyr 1 & $128.7 \mathrm{~s}$ & & $128.5 \mathrm{~s}$ & & $128.7 \mathrm{~s}$ & \\
\hline AcTyr 2,6 & $131.2 \mathrm{dx} 2$ & $6.95 \mathrm{~d}(8.5)$ & $131.1 \mathrm{dx} 2$ & $6.93 \mathrm{~d}(8.5)$ & $131.2 \mathrm{dx} 2$ & $7.03 \mathrm{~d}(8.5)$ \\
\hline AcTyr 3,5 & $115.9 \mathrm{dx} 2$ & $6.72 \mathrm{~d}(8.5)$ & $116.1 \mathrm{dx} 2$ & $6.67 \mathrm{~d}(8.5)$ & $115.9 \mathrm{dx} 2$ & $6.74 \mathrm{~d}(8.5)$ \\
\hline AcTyr 4 & $157.05 \mathrm{~s}$ & & $156.98 \mathrm{~s}$ & & $157.0 \mathrm{~s}$ & \\
\hline AcTyr $\mathrm{OH}$ & & $8.19 \mathrm{~s}$ & & $8.15 \mathrm{~s}$ & & $8.20 \mathrm{~s}$ \\
\hline MePhe NMe & $29.6 \mathrm{q}$ & $2.90 \mathrm{~s}$ & $31.9 \mathrm{q}$ & $2.79 \mathrm{~s}$ & $29.3 \mathrm{q}$ & $2.88 \mathrm{~s}$ \\
\hline MePhe $C=0$ & $167.8 \mathrm{~s}$ & & $167.7 \mathrm{~s}$ & & $166.6 \mathrm{~s}$ & \\
\hline MePhe $\alpha$ & $62.4 \mathrm{~d}$ & $5.46 \mathrm{dd}(8.0,7.2)$ & $59.6 \mathrm{~d}$ & $5.29 \mathrm{dd}(8.5,7.2)$ & $63.2 \mathrm{~d}$ & $5.19 \mathrm{dd}(9.1,5.8)$ \\
\hline MePhe $\beta$ & $35.7 \mathrm{t}$ & $\begin{array}{l}2.50 \mathrm{dd}(13.8,8.0) \\
3.24 \mathrm{dd}(13.8,7.2)\end{array}$ & $33.9 \mathrm{t}$ & $\begin{array}{l}3.03 \text { dd }(14.6,8.5) \\
3.21 \text { dd }(14.6,7.2)\end{array}$ & $34.9 \mathrm{t}$ & $\begin{array}{l}2.71 \mathrm{~m}, \\
3.18 \mathrm{dd}(14.0,5.8)\end{array}$ \\
\hline MePhe 1 & $139.0 \mathrm{~s}$ & & $138.6 \mathrm{~s}$ & & $139.3 \mathrm{~s}$ & \\
\hline MePhe 2,6 & $130.5 \mathrm{dx} 2$ & $7.31 \mathrm{~d}(8.0)$ & $129.9 \mathrm{dx} 2$ & $7.21 \mathrm{~d}(7.6)$ & $130.6 \mathrm{dx} 2$ & $7.38 \mathrm{~d}(8.0)$ \\
\hline MePhe 3,5 & $129.4 \mathrm{dx} 2$ & $7.28 \mathrm{~m}$ & $129.2 \mathrm{dx} 2$ & $7.27 \mathrm{~m}$ & $129.5 \mathrm{dx} 2$ & $7.26 \mathrm{dd}(8.0,8.0)$ \\
\hline $\begin{array}{l}\text { MePhe } 4 \\
\text { dhTra NH }\end{array}$ & $127.4 \mathrm{~d}$ & $\begin{array}{l}7.16 \mathrm{~m} \\
9.19 \mathrm{~d}(9.8)\end{array}$ & $127.2 \mathrm{~d}$ & $\begin{array}{l}7.16 \mathrm{~m} \\
8.52 \mathrm{~d}(10.8)\end{array}$ & $127.4 \mathrm{~d}$ & $\begin{array}{l}7.16 \mathrm{~m} \\
10.14 \mathrm{~d}(10.0)\end{array}$ \\
\hline dhTra $\alpha$ & $119.5 \mathrm{~d}$ & 6.77 dd $(9.8,9.8)$ & $119.5 \mathrm{~d}$ & 6.83 dd $(10.8,9.4)$ & $121.0 \mathrm{~d}$ & 7.47 dd $(14.9,10.0)$ \\
\hline dhTra $\beta$ & $104.4 \mathrm{~d}$ & $5.97 \mathrm{~d}(9.8)$ & $103.0 \mathrm{~d}$ & $6.03 \mathrm{~d}(9.4)$ & $107.7 \mathrm{~d}$ & $6.57 \mathrm{~d}(14.9)$ \\
\hline dhTra 1 & & 10.39 br.s & & 10.49 br.s & & 10.24 br.s \\
\hline dhTra 2 & $125.0 \mathrm{~d}$ & $7.68 \mathrm{~d}(2.5)$ & $123.7 \mathrm{~d}$ & $7.61 \mathrm{~d}(1.9)$ & $123.7 \mathrm{~d}$ & $7.36 \mathrm{~d}(2.5)$ \\
\hline dhTra 3 & $111.1 \mathrm{~s}$ & & $111.5 \mathrm{~s} \mathrm{~d}$ & & $113.6 \mathrm{~s}$ & \\
\hline dhTra 3a & $128.0 \mathrm{~s}$ & & $127.9 \mathrm{~s}$ & & $126.4 \mathrm{~s}$ & \\
\hline dhTra 4 & $119.35 \mathrm{~d}$ & $7.57 \mathrm{~d}(8.0)$ & $119.33 \mathrm{~d}$ & $7.64 \mathrm{~d}(8.0)$ & $120.2 \mathrm{~d}$ & $7.74 \mathrm{~d}(7.7)$ \\
\hline dhTra 5 & $120.2 \mathrm{~d}$ & 7.04 ddd $(8.0,8.0,5.2)$ & $120.3 \mathrm{~d}$ & $7.07 \mathrm{dd}(8.4,8.0)$ & $120.3 \mathrm{~d}$ & $7.07 \mathrm{dd}(7.7,7.7)$ \\
\hline dhTra 6 & $122.6 \mathrm{~d}$ & $7.11 \mathrm{~m}$ & $122.9 \mathrm{~d}$ & $7.16 \mathrm{~m}$ & $122.6 \mathrm{~d}$ & 7.14 dd $(7.7,7.7)$ \\
\hline dhTra 7 & $112.1 \mathrm{~d}$ & $7.38 \mathrm{~m}$ & $112.3 \mathrm{~d}$ & $7.44 \mathrm{~d}(8.0)$ & $112.5 \mathrm{~d}$ & $7.41 \mathrm{~d}(7.7)$ \\
\hline dhTra 7a & $137.0 \mathrm{~s}$ & & $137.1 \mathrm{~s}$ & & $138.2 \mathrm{~s}$ & \\
\hline
\end{tabular}

$a$ : The coupling constants are in parentheses. $\quad b$ : dhTra is $\alpha, \beta$-didehydrotryptamine.

room temperature under room light for one week. Therefore, 1 was suggested to be an isomer of 2 . The ${ }^{13} \mathrm{C}$ NMR spectrum of 1 showed the double number of carbon signals comparing with that of 2 , and the signals of 1 can be separated to two groups in the signal intensity ratio of $5: 4$ by further NMR study (Table 2). ${ }^{1} \mathrm{H}-{ }^{1} \mathrm{H}-\mathrm{COSY}$ and HMBC study deduced the planar structure of the major group of 1 (having larger signals) to be AcPhe-MePhedhTra, which was the same as the structure of 2 (Fig. 4). However, it has $Z$ isomer between $\alpha$ and $\beta$ methines of dhTra, which was revealed by the vicinal coupling constants $\left(J_{\alpha, \beta}=9.6 \mathrm{~Hz}\right)$ and NOEs between dhTra NH $\left(\delta_{\mathrm{H}}\right.$ $9.18) / \mathrm{dhTra} 2\left(\delta_{\mathrm{H}} 7.66\right)$ and dhTra $\beta\left(\delta_{\mathrm{H}} 5.98\right) / \mathrm{dhTra} 4\left(\delta_{\mathrm{H}}\right.$ 7.56). The amide bond between two phenylalanines was revealed to be cis conformer by NOESY experiment, and the chirality of two phenylalanines was both L-form. The planar structure of the minor group of 1 (having smaller signals) was also elucidated as AcPhe-MePhe-dhTra by NMR study, and $Z$ isomer between $\alpha$ and $\beta$ methines of
dhTra was revealed by the vicinal coupling constants $\left(J_{\alpha, \beta}=9.4 \mathrm{~Hz}\right)$ and NOEs between dhTra NH $\left(\delta_{\mathrm{H}} 8.47\right) /$ $\operatorname{dhTra} 2\left(\delta_{\mathrm{H}} 7.58\right)$ and dhTra $\beta\left(\delta_{\mathrm{H}} 6.05\right) / \mathrm{dhTra} 4\left(\delta_{\mathrm{H}} 7.64\right)$. The amide bond between two phenylalanines was revealed to be trans conformer by NOE between AcPhe $\alpha\left(\delta_{\mathrm{H}} 5.06\right)$ and MePhe NMe $\left(\delta_{\mathrm{H}} 2.82\right)$. Consequently, the structure of 1 was elucidated as $N$-acetyl-L-phenylalanyl- $N$-methyl-Lphenylalanyl- $(\alpha Z)-\alpha, \beta$-didehydrotryptamine in which the amide bond between $N$-acetylphenylalanine and $N$ methylphenylalanine is a mixture of cis and trans conformers.

The molecular formula of 4 has one more oxygen than that of 2. NMR analysis of $\mathbf{4}$ comparing $\mathbf{2}$ indicated that $\mathrm{N}$-acetylphenylalanine (AcTyr) of $\mathbf{2}$ is replaced with $\mathrm{N}$-acetyltyrosine in 4 (Fig. 5). Isomerisms of 4 were shown to be cis-amide and ( $\alpha \mathrm{E})$-dhTra. The amino acids were revealed as L-tyrosine and $N$-methyl-L-phenylalanine by chiral HPLC of acid hydrolysate of 4 . Thus, the structure of 4 was elucidated as $N$-acetyl-L-tyrosyl- $N$-methyl-L-phenyl- 
Fig. 3. Selected ${ }^{1} \mathrm{H}-{ }^{1} \mathrm{H}$ COSY, HMBC, and NOESY correlations of 2.

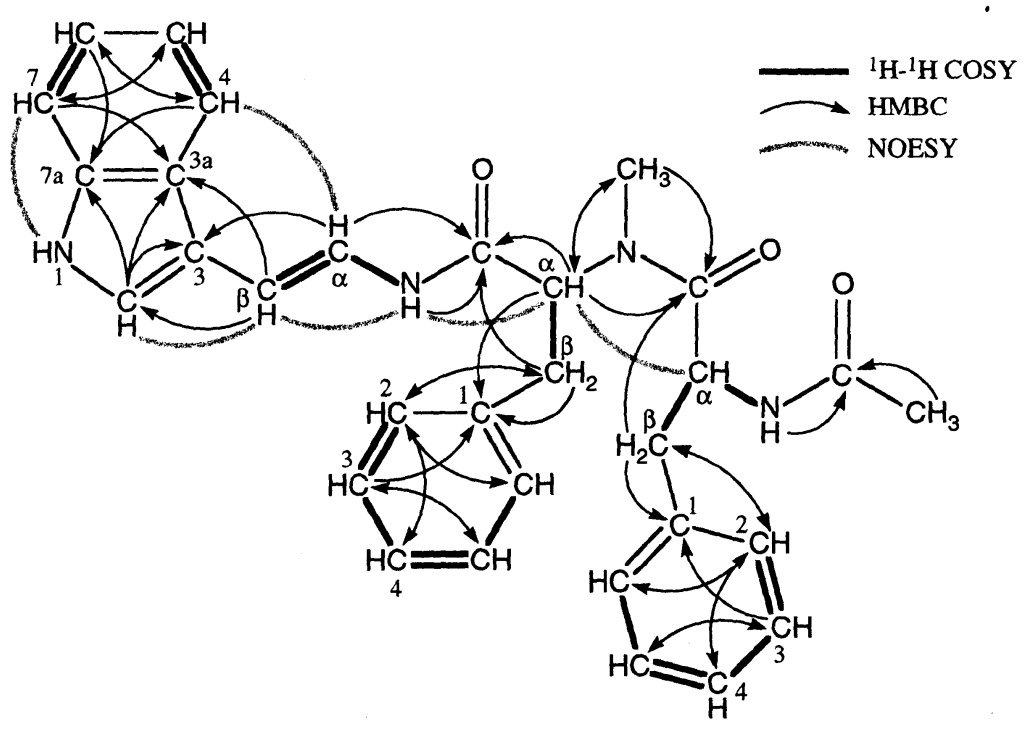

Fig. 4. Selected ${ }^{1} \mathrm{H}-{ }^{1} \mathrm{H}$ COSY, HMBC, and NOESY correlations of $\mathbf{1}$.

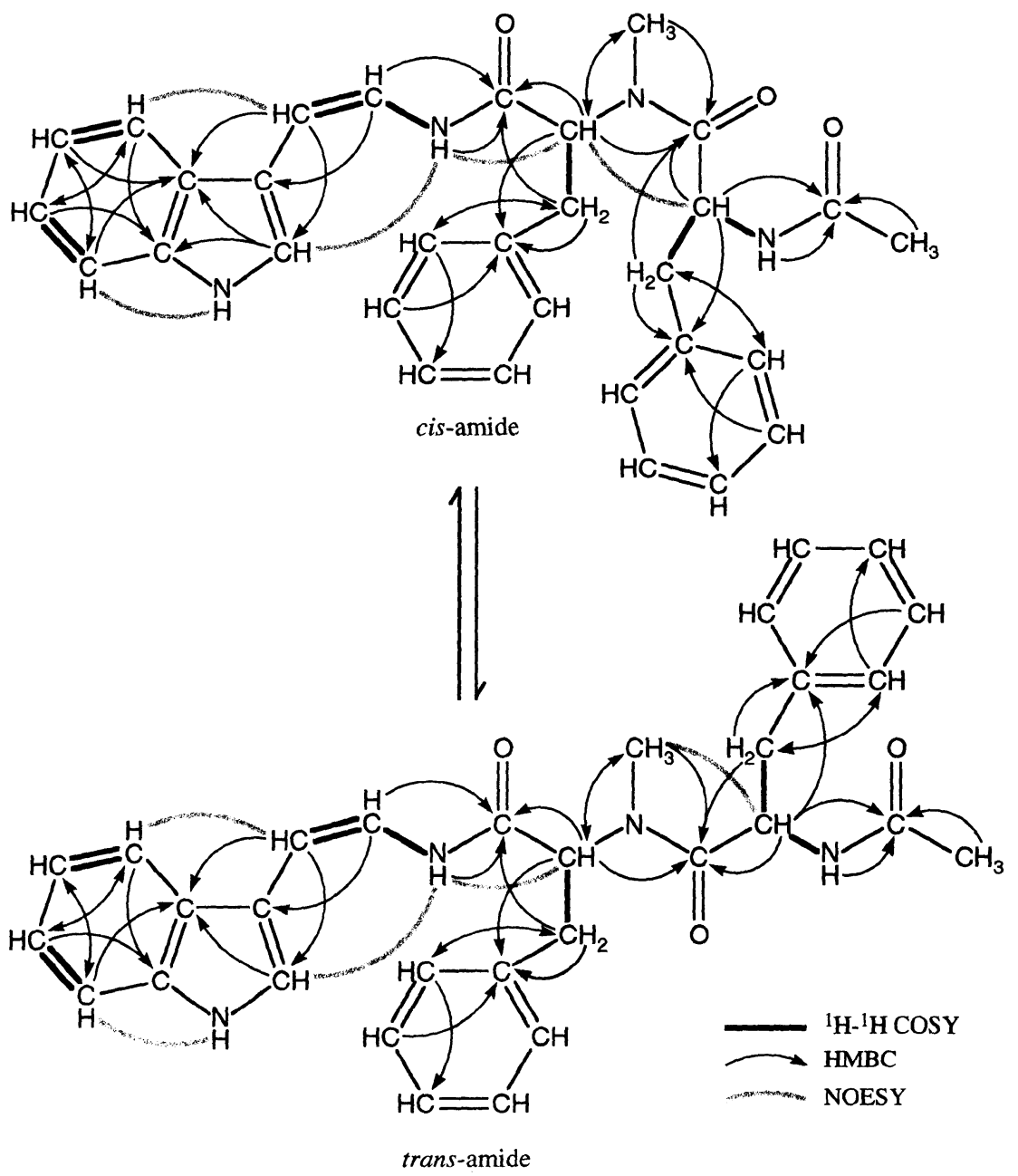


Fig. 5. Selected ${ }^{1} \mathrm{H}-{ }^{1} \mathrm{H}$ COSY, HMBC, and NOESY correlations of $\mathbf{3}$ and $\mathbf{4}$.

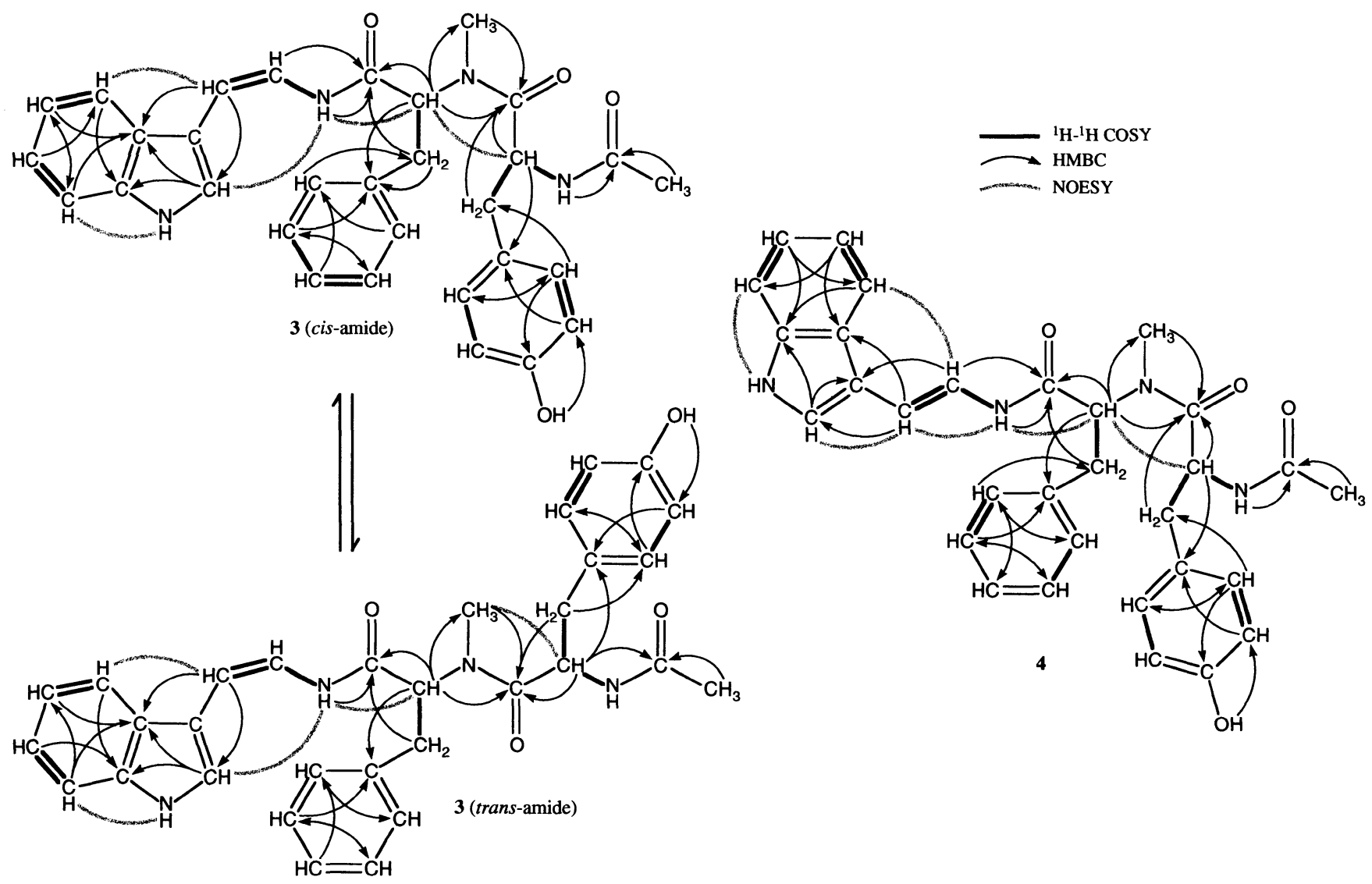

alanyl- $(\alpha E)-\alpha, \beta$-didehydrotryptamine in which the amide bond between $N$-acetyltyrosine and $N$-methylphenylalanine is cis.

The molecular formula of $\mathbf{3}$ was the same as that of $\mathbf{4}$, and more than $90 \%$ of $\mathbf{3}$ was converted to $\mathbf{4}$ in methanol for one week (room temperature under room light). The ${ }^{13} \mathrm{C}$ NMR spectrum of $\mathbf{3}$ showed a double number of carbon signals comparing with that of 4 . NMR signals of 3 can be also separated to two groups in the signal intensity ratio of $5: 4$. Therefore, 3 was suggested to be $(\alpha Z)$-dhTra isomer of 4 by analogy with the conversion from 1 to 2 . Further NMR analysis (Fig. 5) revealed that $\mathbf{3}$ is $N$-acetyl-L-tyrosyl$N$-methyl-L-phenylalanyl- $(\alpha Z)-\alpha, \beta$-didehydrotryptamine in which the amide bond between $N$-acetyltyrosine and $N$-methylphenylalanine is a mixture of cis and trans conformers.

Miyakamides have a common structure of dipeptidyl$\alpha, \beta$-didehydrotryptamine. $l$-Phenylalaninamide, terpeptin, and aspergillamides $\mathrm{A}$ and $\mathrm{B}$ belong to the same group, and they are all produced by Aspergillus spp. ${ }^{12 \sim 14)}$ Similar compounds have been also isolated from, Penicillium sp. (penidiamide), red algae (fragilamide, chondriamides $\mathrm{A} \sim$ $\mathrm{C}$ ), and invertebrates (halocyamines $\mathrm{A}$ and $\mathrm{B}$, coscinamides $\mathrm{A} \sim \mathrm{C}) .{ }^{15 \sim 20)}$ Among them, aspergillamides $\mathrm{A}$ and $\mathrm{B}$ are closely related to miyakamides. Their planar structure is AcLeu-MePhe-dhTra, and isomerisms of aspergillamides A and $\mathrm{B}$ are $(\alpha Z)$-dhTra with cis- and trans-amide mixture and $(\alpha E)$-dhTra with cis-amide, respectively. It is interesting that $l$-phenylalaninamide, terpeptin, and miyakamides were all isolated from Aspergillus spp. collected from soil samples in Ryukyu Islands, Japan. Aspergillamides producing strain was collected in Acklins Island, the Bahamas, and most other $\alpha, \beta$-didehydrotryptamine containing compounds were isolated from marine algae or marine invertebrates. Therefore, it is suggested that the producing organisms of $\alpha, \beta$-didehydrotryptamine containing compounds are related to the marine environment. 
Table 4. Antimicrobial spectra of $\mathbf{1} \sim \mathbf{4}$.

\begin{tabular}{lrrrc}
\hline \multirow{2}{*}{ Test microorganism } & \multicolumn{4}{c}{ MIC $(\mu \mathrm{g} / \mathrm{ml})$} \\
& $\mathbf{1}$ & $\mathbf{2}$ & $\mathbf{3}$ & $\mathbf{4}$ \\
\hline Staphylococcus aureus ATCC6538p & $>100$ & $>100$ & $>50$ & $>50$ \\
Bacillus subtilis ATCC6633 & $>100$ & $>100$ & $>50$ & $>50$ \\
Micrococcus luteus ATCC9341 & $>100$ & $>100$ & - & - \\
Mycobacterium smegmatis ATCC607 & $>100$ & $>100$ & - & - \\
Escherichia coli NIHJ & $>100$ & $>100$ & $>50$ & $>50$ \\
Pseudomonas aeruginosa IFO3080 & $>100$ & $>100$ & $>50$ & $>50$ \\
Xanthomonas campestris pv. oryzae KB88 & 100 & 100 & - & - \\
Candida albicans KF1 & $>100$ & $>100$ & $>50$ & $>50$ \\
Saccharomyces cerevisiae KF26 & $>100$ & $>100$ & $>50$ & $>50$ \\
Aspergillus niger ATCC6275 & $>50$ & 50 & - & - \\
Mucor racemosus IFO4581 & $>100$ & $>50$ & $>50$ & $>50$ \\
\hline
\end{tabular}

-, not tested

\section{Biological Activities}

Insecticidal and nematocidal activities of miyakamides were studied by a microplate assay using brine shrimp Artemia salina and free-living nematode Caenorhabditis elegans. The assay method was reported previously. ${ }^{1,5)}$ Minimum growth inhibitory concentrations of $\mathbf{1}$ and $\mathbf{2}$ against $A$. salina were $5 \mu \mathrm{g} / \mathrm{ml}$, and those of $\mathbf{3}$ and 4 were $20 \mu \mathrm{g} / \mathrm{ml}$. However, all miyakamides did not affect C. elegans at $100 \mu \mathrm{g} / \mathrm{ml}$. Antimicrobial activities of miyakamides are shown in Table 4. Compounds 1 and $\mathbf{2}$ showed weak activity against Xanthomonas campestris, but 3 and 4 did not show any antimicrobial activities. The $\mathrm{IC}_{50}$ values of 1, 2, 3, and 4 against P388 cells were 10.5, 12.2, 8.8 , and $7.6 \mu \mathrm{g} / \mathrm{ml}$, respectively. Though anti- $A$. salina activities of $\mathbf{1}$ and $\mathbf{2}$ were more potent than those of $\mathbf{3}$ and $\mathbf{4}$, their cytotoxicities against $\mathrm{P} 388$ cells were similar.

\section{Experimental}

\section{General}

Morphological observations of the miyakamides producing strain were carried out using an Olympus VanoxS AH-2 microscope and a JEOL JSM-5600 scanning electron microscope.

NMR spectra were recorded on a Varian Inova 600 spectrometer $\left({ }^{2-3} J_{\mathrm{CH}}=8 \mathrm{~Hz}\right.$ in $\left.\mathrm{HMBC}\right)$. Chemical shifts are shown in $\delta$ values (ppm) relative to acetone- $d_{6}$ at $2.06 \mathrm{ppm}$ for ${ }^{1} \mathrm{H}$ NMR and at $29.8 \mathrm{ppm}$ for ${ }^{13} \mathrm{C}$ NMR. Mass spectrometry was conducted on a JEOL JMS-AX505 HA spectrometer. UV and IR spectra were measured with a Shimadzu UV-240 spectrophotometer and a Horiba FT-210 Fourier transform infrared spectrometer, respectively. Optical rotations were recorded on a JASCO model DIP181 polarimeter. Melting points were measured with a Yanaco micro melting point apparatus MP-S3.

\section{Taxonomic Studies of the Producing Organism}

The strain FKI-0739 was isolated from a fallen leaf collected at Miyakojima Island, Japan. Taxonomic studies and identification were conducted according to the procedure described by KLICK and PITT, ${ }^{6)}$ and RAPER and FENNEL. ${ }^{7)}$ Morphological observations were done under a microscope (Olympus Vanox-S AH-2) and a scanning electron microscope (JEOL JSM-5600). Color hues were described according to Color Harmony Manual, 4th Ed. ${ }^{21)}$

\section{Media}

The seed medium was composed of $2.4 \%$ glucose, $0.5 \%$ Polypepton (Nihon Pharmaceutical Co.), $0.5 \%$ yeast extract (Oriental Yeast Co.), $0.4 \% \mathrm{MgSO}_{4} \cdot 7 \mathrm{H}_{2} \mathrm{O}, 0.1 \% \mathrm{KH}_{2} \mathrm{PO}_{4}$, and $0.1 \%$ agar ( $\mathrm{pH} 5.6$ prior to sterilization). The production medium was composed of $2.0 \%$ glycerol, $1.0 \%$ sucrose, $0.5 \%$ ammonium acetate, $0.2 \%$ Cultivator \#100 (fish extract, Yaizu Suisankagaku Industry Co.), 0.1\% agar, $0.1 \% \mathrm{~K}_{2} \mathrm{HPO}_{4}, 0.05 \% \mathrm{MgSO}_{4} \cdot 7 \mathrm{H}_{2} \mathrm{O}$, and $0.05 \% \mathrm{KCl}(\mathrm{pH}$ 6.0 prior to sterilization). 
Hydroxyaspergillic Acid (6)

FAB-MS $(m / z) 503(2 \mathrm{M}+\mathrm{Na})^{+}, 765(3 \mathrm{M}+2 \mathrm{Na}-\mathrm{H})^{+}$; HR-FAB-MS $(\mathrm{m} / \mathrm{z})$ found $503.2759(2 \mathrm{M}+\mathrm{Na})^{+}$, calcd 503.2845 (for $\mathrm{C}_{24} \mathrm{H}_{40} \mathrm{~N}_{4} \mathrm{O}_{6} \mathrm{Na}$ ); ${ }^{1} \mathrm{H} \quad \mathrm{NMR} \quad(600 \mathrm{MHz}$, acetone- $\left.d_{6}\right) \delta 7.50 \mathrm{~s}(1 \mathrm{H}, 6-\mathrm{H}), 6.73$ br.s $(1 \mathrm{H}, 4-\mathrm{OH}), 2.56$ m $\left(2 \mathrm{H}, \mathrm{CH}_{2} \mathrm{CH}\left(\mathrm{CH}_{3}\right)_{2}\right), 2.12 \mathrm{~m}\left(1 \mathrm{H}, \mathrm{CH}\left(\mathrm{CH}_{3}\right)_{2}\right), 1.94 \mathrm{~m}$ $\left(2 \mathrm{H}, \mathrm{CH}_{2} \mathrm{CH}_{3}\right), 1.50 \mathrm{~s}\left(3 \mathrm{H}, \mathrm{C}\left(\mathrm{CH}_{3}\right) \mathrm{OH}\right), 0.85 \mathrm{~d}(6 \mathrm{H}$, $\left.J=6.3 \mathrm{~Hz}, \mathrm{CH}\left(\mathrm{CH}_{3}\right)_{2}\right), 0.81 \mathrm{t}\left(3 \mathrm{H}, J=7.3 \mathrm{~Hz}, \mathrm{CH}_{2} \mathrm{CH}_{3}\right) ;{ }^{13} \mathrm{C}$ NMR ( $125 \mathrm{MHz}$, acetone- $\left.d_{6}\right) \delta 158.3 \mathrm{~s}(\mathrm{C}-3), 150.5 \mathrm{~s}(\mathrm{C}-$

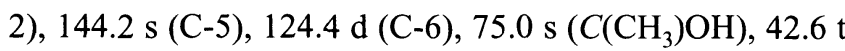
$\left(\mathrm{CH}_{2} \mathrm{CH}\left(\mathrm{CH}_{3}\right)_{2}\right), 33.2 \mathrm{t}\left(\mathrm{CH}_{2} \mathrm{CH}_{3}\right), 28.6 \mathrm{~d}\left(\mathrm{CH}\left(\mathrm{CH}_{3}\right)_{2}\right), 24.8$

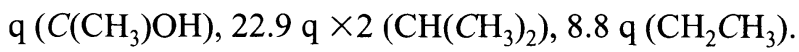

\section{Acid Hydrolysis of Miyakamides}

Each miyakamide $(100 \mu \mathrm{g})$ was hydrolyzed with $6 \mathrm{~N} \mathrm{HCl}$ $(990 \mu \mathrm{l})-1 \%$ phenol $(10 \mu \mathrm{l})$ vapor at $150^{\circ} \mathrm{C}$ for 5 hours by Pico-Tag Workstation (Waters). The reaction mixture was concentrated to dryness, dissolved in a small amount of water, and charged on a chiral HPLC: column, Sumichiral OA-5000 (Sumika Chemical Analysis Service, i.d. 4.6× $150 \mathrm{~mm}$ ); mobile phase, $2 \mathrm{~mm} \mathrm{CuSO}_{4}-\mathrm{MeOH}$ (85:15); flow rate $1.0 \mathrm{ml} /$ minute; detection, UV $254 \mathrm{~nm}$. The amino acids were identified by comparison with each authentic optically pure sample. Authentic L-phenylalanine, D-phenylalanine, $N$-methyl-L-phenylalanine, $N$-methyl-D-phenylalanine, L-tyrosine, and D-tyrosine were eluted at 45.1, 62.0, $39.8,54.1,16.3$, and 22.3 minutes, respectively.

\section{Conversion of 1 to 2 and 3 to 4}

Compound 1 or $3(1 \mathrm{mg})$ was dissolved in $1 \mathrm{ml}$ of $\mathrm{MeOH}$ in a glass test tube and kept at room temperature under room light for one week. Then the solution was analyzed by HPLC.

\section{Acknowledgements}

We are grateful to Ms. Hiroko HaTANO, Kitasato Institute for Life Sciences, Kitasato University for measurements of NMR spectra. The authors also thank Ms. AKIKO NAKAGAWA and Ms. CHIKAKo SAKABE of School of Pharmaceutical Sciences, Kitasato University for measurements of mass spectra. The financial support by Japan Keirin Association is also gratefully acknowledged. KS is supported partly by hohansya foundation.

\section{References}

1) Ōmura, S.; Y. Enomoto, M. Shinose, Y. TAKahashi, Y. IWAI \& K. SHIOMI: Isolation and structure of a new antibiotic viridomycin F produced by Streptomyces sp. K96-0188. J. Antibiotics 52: 61 64, 1999

2) Ōmura, S.; N. Arai, Y. Yamaguchi, R. Masuma, Y. Iwai, M. Namikoshi, A. Turberg, H. Kölbl \& K.
SHIOMI: Argifin, a new chitinase inhibitor, produced by Gliocladium sp. FTD-0668 I. Taxonomy, fermentation, and biological activities. J. Antibiotics 53: 603 608, 2000

3) Arai, N.; K. Shiomi, Y. Yamaguchi, R. Masuma, Y. IwaI, A. Turberg, H. KÖlbl \& S. ŌMura: Argadin, a new chitinase inhibitor, produced by Clonostachys sp. FO-7314. Chem. Pharm. Bull. 48: 1442 1446, 2000

4) Ōmura, S.; H. Miyadera, H. Ui, K. Shiomi, Y. Yamaguchi, R. Masuma, T. Nagamitsu, D. Takano, T. Sunazuka, A. Harder, H. Kölbl, M. NAMikoshi, H. Miyoshi, K. Sakamoto \& K. Kita: An anthelmintic compound, nafuredin, shows selective inhibition of complex I in helminth mitochondria. Proc. Natl. Acad. Sci. U.S.A. 98: 60 62, 2001

5) Enomoto, Y.; K. Shiomi, A. Matsumoto, Y. Takahashi, Y. Iwai, A. Harder, H. KÖlbl, H. B. WoOdRufF \& S. ŌMURA: Isolation of a new antibiotic oligomycin $G$ produced by Streptomyces sp. WK-6150. J. Antibiotics 54: 308 313, 2001

6) Klich, M. A. \& J. I. PItT: A laboratory guide to the common Aspergillus species and their teleomorphs. CSIRO, North Ryde, 1988

7) PAPer, K. B. \& D. I. Fennell: The genus Aspergillus p. 686, The Williams and Wilkins, Baltimore, 1965

8) Hamasaki, T.; H. Kuwano, K. Isono, Y. Hatsuda, K. FukuYama, T. TSUKinara \& Y. Katsube: A new metabolite, parasiticolide A, from Aspergillus parasiticus. Agric. Biol. Chem. 39: 749 751, 1975

9) Gould, R. O.; T. J. Simpson \& M. D. WALKINSHAw: Isolation and X-ray crystal structures of astellolides $\mathrm{A}$ and B, sesquiterpenoid metabolites of Aspergillus variecolor. Tetrahedron Lett. 22: 1047 1050, 1981

10) Dutcher, J. D.: Aspergillic acid: an antibiotic substance produced by Aspergillus flavus. III. Structure of hydroxyaspergillic acid. J. Biol. Chem. 232: 785 795, 1958

11) YABUTA, T.: A new organic acid (kojic acid) formed by Aspergillus oryzae. J. Chem. Soc. Japan 37: 1185 1233, 1916

12) NaKano, Y. \& M. Sugawara (Otsuka Pharmaceutical Co., Ltd.): Fermentative manufacture of indoles. Jpn. Kokai 01-149766, June 12, 1989

13) Kagamizono, T.; N. SaKai, K. Arai, K. Kobinata \& H. OsADA: Terpeptin, a novel mammalian cell cycle inhibitor, produced by Aspergillus terreus 95F-1. Tetrahedron Lett. 38: 1223 1226, 1997

14) Toske, S. G.; P. R. Jensen, C. A. Kauffman \& W. FENICAL: Aspergillamides A and B: modified cytotoxic tripeptides produced by a marine fungus of the genus Aspergillus. Tetrahedron 54: 13459 13466, 1998

15) Witter, L.; T. ANKE \& O. Sterner: A new inhibitor of synovial phospholipase $\mathrm{A}_{2}$ from fermentations of Penicillium sp. 62-92. Z. Naturforsch., C: Biosci. 53: 60 64, 1998

16) Kirkup, M. P. \& R. E. Moore: Indole alkaloids from the marine red alga Martensia fragilis. Tetrahedron Lett. 24: 2087 2090, 1983

17) Palermo, J. A.; P. B. Flower \& A. M. Seldes: Chondriamides $\mathrm{A}$ and $\mathrm{B}$, new indolic metabolites from the red alga Chondria sp. Tetrahedron Lett. 33: 3097 3100, 1992

18) Davyt, D.; W. Entz, R. Fernandez, R. Mariezcurrena, 
A. W. Mombru, J. Saldana, L. Dominguez, J. Coll \& E. MANTA: A new indole derivative from the red alga Chondria atropurpurea. Isolation, structure determination, and anthelmintic activity. J. Nat. Prod. 61: 1560 1563, 1998

19) Azumi, K.; H. Yokosawa \& S. IsHiI: Halocyamines: novel antimicrobial tetrapeptide-like substances isolated from the hemocytes of the solitary ascidian Halocynthia roretzi. Biochemistry 29: 159 165, 1990

20) Bokesch, H. R.; L. K. Pannell, T. C. McKee \& M. R. BoyD: Coscinamides $\mathrm{A}, \mathrm{B}$ and $\mathrm{C}$, three new bis indole alkaloids from the marine sponge Coscinoderma $\mathrm{sp}$. Tetrahedron Lett. 41: 6305 6308, 2000

21) Jacobson, E.; W. C. Granville \& C. E. Foss: Color Harmony Manual 4th Ed. Container of America, Chicago, 1958 